\title{
Conocimientos, actitudes y percepciones de madres sobre la alimentación de niños menores de 3 años en una comunidad de Lima
}

\author{
Mothers' knowledge, attitudes and perceptions on children less than 3 year-old nutrition in \\ a Lima's community
}

\author{
Raquel Gamarra-Atero ${ }^{1 a}$, Mayra Porroa-Jacobo ${ }^{1 a}$, Margot Quintana-Salinas ${ }^{2 b}$ \\ ${ }^{1}$ Escuela Académico Profesional de Nutrición, Facultad de Medicina, UNMSM. Lima, Perú. \\ 2 Departamento de Nutrición, Facultad de Medicina, UNMSM. Lima, Perú. \\ a Alumna, ${ }^{\text {b Docente }}$
}

\section{Resumen}

Introducción: Para lograr una adopción de conducta, se precisa identificar los factores internos que la afectan. Objetivos: Identificar conocimientos, actitudes y percepciones de madres sobre alimentación del niño menor de 3 años. Diseño: Estudio observacional descriptivo, cuanticualitativo. Lugar: Asentamientos Humanos del distrito de Villa María del Triunfo- Lima. Participantes: Madres de niños menores de 3 años. Intervenciones: Previo consentimiento, para la fase cuantitativa se aplicó un cuestionario de 10 preguntas cerradas con respuestas dicotómicas sobre conocimientos y 10 preguntas con categorías de respuesta en escala de Likert sobre actitudes en 161 madres, y para la fase cualitativa se realizó 28 entrevistas a profundidad y 4 grupos focales para identificar percepciones en 68 madres. Principales medidas de resultados: Nivel de conocimientos, tipo de actitudes y percepciones sobre lactancia materna (LM), alimentación complementaria $(\mathrm{AC})$ y alimentación durante una enfermedad. Resultados: $81 \%$ de participantes mostró nivel de conocimientos alto, $65 \%$ estuvo en total acuerdo con la LM exclusiva durante los 6 primeros meses de vida, $63 \%$ estaba de acuerdo con prácticas adecuadas en alimentación complementaria. La mayoría percibía que la LM debía ser hasta dos años, porque ayuda a la formación de órganos y para que el niño crezca sano; la $A C$ se inicia a los seis meses, porque ayuda a la formación del estómago, el niño tiene más apetito y la LM ya no es suficiente; el consumo de alimentos de origen animal, frutas y verduras debía ser diario; durante la diarrea se brindaría abundante líquidos y mayor frecuencia de alimentos y en menor cantidad. Conclusiones: Las madres presentaron nivel de conocimientos alto $(81 \%)$ y actitudes favorables $(66,7 \%)$ sobre alimentación infantil, y sus percepciones en general coincidían con ello.

Palabras clave: Conocimiento; actitud; percepción; alimentación; niño.
Abstract

Introduction: To adopt a conduct it is necessary to identify internal factors that affect it. Objectives: To identify mothers' knowledge, attitudes and perceptions on children less than 3 year-old nutrition. Design: Quantitative qualitative study, descriptive, exploratory. Location: Villa Maria del Triunfo Human Settlements, Lima, Peru. Participants: Mothers of children less than 3 year-old. Interventions: Following informed consent, for the quantitative phase phase we applied a questionnaire of 10 closed questions with dichotomous responses on knowledge and 10 questions with Likert's response categories on attitudes to 161 mothers; and for the qualitative we did 28 depth interviews and 4 focus groups to identify perceptions were conducted to 68 mothers. Main outcome measures: Level of knowledge, type of attitudes and perceptions on breastfeeding (BF), complementary feeding (CF) and feeding during illness. Results: Eighty-one per cent of participants showed high level of knowledge, $65 \%$ showed complete agreement with exclusive BF during the first 6 months, $63 \%$ agreed with adequate complementary feeding practices. Most felt that BF should be offered up to 2 years because it helps organs development and the children to grow up healthy. CF should be started at six months of age because it helps the stomach function, the child has more appetite and BF is no longer sufficient; consumption of animal foods, fruits and vegetables should be daily; during diarrhea plenty of fluids and more frequent food and in smaller amounts should be provided. Conclusions: Mothers showed high levels of knowledge $(81 \%)$, favorable attitudes $(66,7 \%)$ on infant feeding and their perceptions in general agreed with it.

Key words: Knowledge; attitude; perception; feeding; child.

An Fac med. 2010;71(3):179-83

\section{INTRODUCCIÓN}

Una adecuada alimentación durante los primeros años de vida es fundamental para asegurar el crecimiento y mantener la salud durante la etapa adulta ${ }^{(1,2)}$. A partir de los 6 meses de vida se debe iniciar una etapa conocida como alimentación complementaria (AC), donde se introduce alimentos diferentes a la leche materna de manera gradual y progresiva ${ }^{(3-5)}$. La AC tiene por objetivo cubrir las necesidades nutricionales del niño, favorecer la interrelación madre-hijo, ayudar a la transición del lactante de una dieta líquida a la del consumo familiar y favorecer el establecimiento de hábitos saludables de alimentación. Para lograr lo anterior es importante que la madre tenga conocimientos y prácticas adecuadas en alimentación.

Por otro lado, las actitudes, definidas como estado de disposición psicológica, adquirida y organizada a través de la experiencia que incita a reaccionar de una manera característica frente a determinadas personas, objetos o situaciones ${ }^{(6)}$, pueden ser favorables o desfavorables, de rechazo o aceptación respecto a lactancia materna (LM), alimentación habitual del niño menor de 3 años y durante periodos de enfermedad. Asimismo, las percepciones (procesos cognitivos que consisten en el reconocimiento, interpretación y significación para elaborar juicios en torno a las sensaciones obtenidas del ambiente físico y social) (7) sobre la alimentación del niño, creencias en relación a LM, consistencia de las comidas, cantidad, frecuencia y consumo de alimentos de origen animal (AOA), influirían en sus actitudes ${ }^{(8)}$. 
La Encuesta Demográfica y de Salud Familiar (ENDES) Continua 2007-2008 revela que $64,3 \%$ de niños menores de 3 años consume granos y cereales, $39,3 \%$ no consume AOA y más de $70 \%$ no consume menestras ${ }^{(9)}$. Esto refleja que las madres brindan a sus niños una alimentación basada principalmente en alimentos energéticos y no balanceada, lo cual hace suponer que no tendrían adecuados conocimientos acerca de alimentación. Similar situación se observa en las comunidades del área de intervención del Programa de Desarrollo del Área (PDA) 'El Salvador', zona de intervención de la ONG World Vision International (WVI), en el distrito de Villa María del Triunfo (VMT), Lima, cuya línea de base del 2007 mostró que $84 \%$ de las madres de niños menores de 5 años les proporciona una alimentación inadecuada ${ }^{(10)}$, definiendo inadecuada como el no suministro de al menos un alimento de cada grupo (energéticos, constructores y reguladores) en cada una sus comidas, lo que nos llevaría a cuestionar las prácticas y, por ende, los conocimientos, que tendrían las madres de esta zona. Según un estudio en Villa El Salvador, distrito aledaño a VMT, $1,8 \%$ de las madres mostró un nivel de conocimiento regular sobre alimentación infantil $^{(11)}$.

Actualmente, la información acerca de actitudes y percepciones de las madres con respecto a la alimentación de sus niños durante los 3 primeros años de vida es limitada, por lo que se conoce poco sobre por qué realizan ciertas prácticas y por qué no otras, siendo esta información la que nos ayudaría a darle un enfoque real y útil a las intervenciones educativonutricionales que se realizan en busca de una mejora del estado de salud y nutrición de los niños.

En Costa Rica, un estudio cualicuantitativo halló que más de la mitad de madres percibía que los $\mathrm{AOA}$ eran de alto valor nutritivo y menos de la mitad reconoció a los cereales, leguminosas, hortalizas y frutas como más nutritivos para el niño. También percibían que ciertos alimentos, como los frijoles y las comidas grasosas o preparaciones con carne de cerdo, eran perjudiciales en la alimentación del niño menor de un año ${ }^{(12)}$.
El PDA 'El Salvador' es una institución que realiza constantemente intervenciones sobre temas de salud y nutrición en zonas urbano marginales de nuestro país. Por ello, es necesario contar con información que permita orientar mejor su intervención educativa para lograr la adopción de conductas positivas. Así, el objetivo del presente estudio fue identificar el nivel de conocimientos, las actitudes y percepciones sobre la alimentación del niño menor de 3 años en madres de una comunidad de nuestra capital.

\section{MÉTODOS}

El presente trabajo tuvo un enfoque cuanticualitativo y de tipo descriptivo exploratorio. En la fase cuantitativa se identificaron los conocimientos y actitudes sobre alimentación del niño en 161 madres de niños menores de 3 años de 6 asentamientos humanos (AA HH) de la zona 2 del PDA 'El Salvador', en el distrito de Villa María del Triunfo, durante el año 2009. El muestreo fue no probabilístico por conveniencia.

Se elaboró un cuestionario de 2 segmentos: conocimientos, constituido por 10 preguntas cerradas con respuestas dicotómicas; y actitudes, constituido por 10 afirmaciones correctas evaluadas según escala de Likert. Ambos contaban con preguntas sobre LM, AC y alimentación durante un episodio de enfermedad. Este instrumento fue validado por juicio de expertos y por un piloto aplicado en madres de la comunidad no participantes del estudio.

Previo a la captación de información, se ubicó la dirección de las familias del padrón del censo comunitario proporcionado por el Proyecto Salud y Nutrición del PDA El Salvador. Identificadas las madres, se las visitó para solicitar su consentimiento y aplicar el cuestionario. Posteriormente, los cuestionarios fueron revisados, obteniéndose 158 correctamente llenados. Para la calificación del segmento de conocimientos se asignó 2 puntos a cada pregunta, siendo el puntaje máximo alcanzable 20 puntos, teniendo como puntos de corte: Bajo de 0 a 6, Re- gular de 7 a 13 y Alto de 14 a 20. Mientras que para las actitudes, cada ítem se evaluó según una escala de 5 alternativas: Totalmente en desacuerdo, En desacuerdo, $\mathrm{Ni}$ en acuerdo ni en desacuerdo, De acuerdo, y Totalmente de acuerdo, cuyo valor iba de 1 a 5 . Así, el puntaje máximo alcanzable era 50 puntos. Se consideró que de 25 a menos la madre tenía una Actitud desfavorable y de 26 a 50, una Actitud favorable. Una vez obtenida la información se procedió a digitarla y codificarla en Microsoft Excel 2007, para luego ser presentada en porcentajes.

En la fase cualitativa, se identificaron las percepciones sobre los mismos temas de la fase cuantitativa, en 68 madres seleccionadas según criterios socioestructurales: Rango de edad (18 a 25; 26 a 35; 36 a 45 años), nivel educativo (primaria, secundaria, superior) y condición materna (primíparas, multíparas), fueron captadas intencionalmente. Participaron 28 madres para la entrevista a profundidad y 40 para los grupos focales. Se aplicó ambas técnicas para triangular la información, elaborándose para cada técnica una guía de preguntas semejantes que respondieran a los objetivos del estudio, para asegurar la confiabilidad de los datos. Con las madres identificadas y previo consentimiento, se realizaron visitas a domicilio para efectuar la entrevista, que fue grabada para el análisis de la información. Se realizó un grupo focal en 4 AA HH, ya que a 2 de ellos acudieron madres de otros 2 AA HH aledaños; se contó para cada uno con 1 moderador, 2 observadores y 1 grabadora de voz. En ambas técnicas se utilizó una bitácora de campo. La información de ambas técnicas fue degrabada y analizada según su contenido, por cada investigador. Se elaboró una primera matriz, donde las transcripciones fueron agrupadas según ítem evaluado y técnica utilizada. Posteriormente, se elaboró una segunda matriz, donde se seleccionó las transcripciones con contenidos semejantes y se asignó etiquetas, se interpretó los datos y se llegó a un consenso para cada ítem, lo que contribuyó a establecer el argumento correspondiente. 


\section{RESULTADOS}

La muestra estuvo formada por $158 \mathrm{ma}$ dres de niños menores de 3 años residentes en la zona 2 del área de intervención del PDA 'El Salvador', en el distrito de Villa María del Triunfo. Las participantes presentaban entre 18 a 42 años, con una mediana de edad de 30 años; $60 \%$ tenía nivel educativo secundario, seguido de $29 \%$ con nivel educativo primario, mientras $9 \%$ tuvo nivel educativo superior; se resalta que $2 \%$ era analfabeta.

El 81\% presentó un nivel de conocimientos alto, $17 \%$ nivel regular y $2 \%$ nivel bajo. La mayoría de madres respondió correctamente sobre la duración de la LME (82\%). Así mismo, la mayoría respondió adecuadamente sobre AC. Sin embargo, los porcentajes más bajos de respuestas correctas fueron para el consumo diario de AOA y para el reconocimiento del segundo como mejor alimento en comparación a la sopa. Contrariamente, la pregunta con mayor número de respuestas correctas fue la edad de inicio de la AC (97\%) (figura 1). El 53,3\% respondió inadecuadamente al aumento del número de comidas después de un episodio de enfermedad y a brindar comidas espesas durante episodios de diarrea.

El $66,7 \%$ mostró una actitud favorable hacia las características de la alimentación del niño menor de 3 años y $29,8 \%$ no estuvo ni en acuerdo ni en desacuerdo con el tema. El 77\% se mostró de acuerdo en que la LM debe ser exclusiva durante los 6 primeros meses de vida. Sin embargo, fue preocupante observar que 14\% aún se muestra en desacuerdo con esta afirmación, por varias razones. La mayoría de madres dijo estar de acuerdo con las prácticas adecuadas durante la AC (63\%), definidas como brindar preparaciones espesas, agregar grasa (aceite o mantequilla), número de comidas, consumo diario de $\mathrm{AOA}$, frutas y verduras, mientras que $20 \%$ no estuvo ni en acuerdo ni en desacuerdo. De igual forma, la mayoría dijo estar de acuerdo con las prácticas adecuadas durante y después de un episodio de enfermedad (41\%); sin embargo, $25 \%$ se mostró en desacuerdo con respecto a estas, siendo similar la cifra para aquellas que no se mostraron ni en acuerdo ni en desacuerdo con estas prácticas (24\%) (figura 2).

En relación a las percepciones, la mayoría reconoció que la LM debe durar hasta los 2 años, principalmente porque ayuda en la formación de órganos, el bajo consumo de alimentos antes de esa edad y su aporte para que el niño crezca sano. Sin embargo, algunas madres percibieron que la LM debe ser retirada antes de los 2 años para permitir el consumo de otros alimentos, para que la madre trabaje y si la madre siente dolor cuando da de lactar.

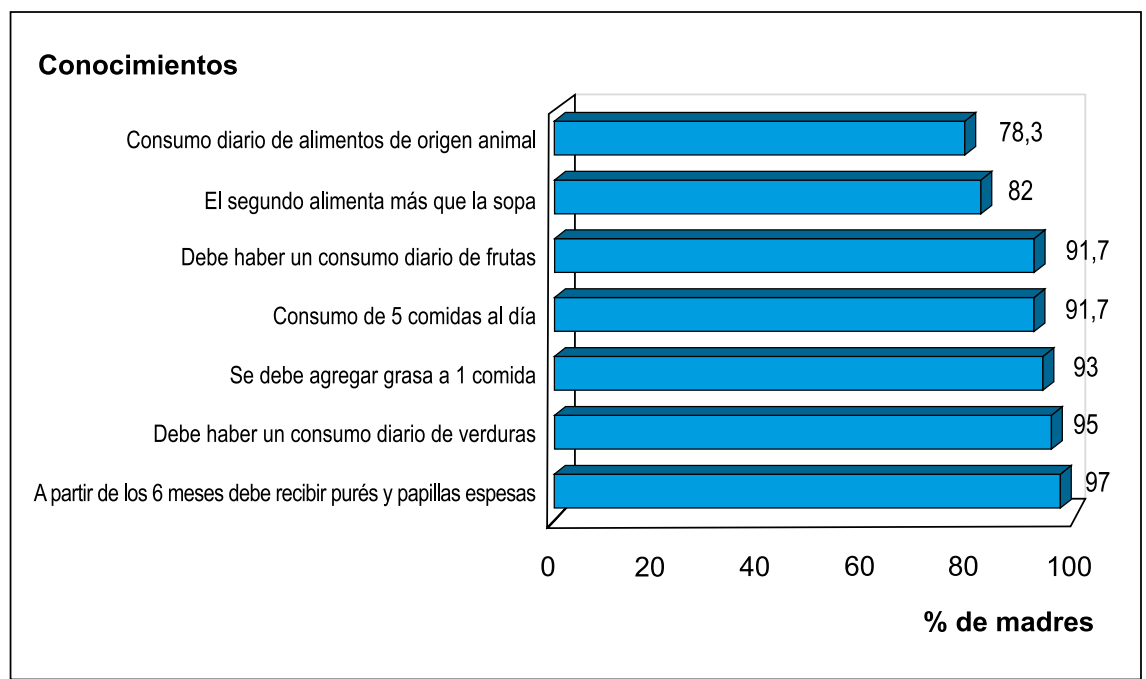

Figura 1. Distribución de madres que respondieron adecuadamente las preguntas sobre alimentación complementaria.
Con respecto al inicio de la $\mathrm{AC}$ a los 6 meses, la mayoría refirió que era porque el niño requiere más alimentos a partir de esa edad, y ayuda a la formación del estómago, tiene más apetito y porque cambia la composición de la leche materna. Otro grupo refirió que se debe iniciar antes porque 'el niño se antoja' y a esa edad ya puede comer con normalidad. Además, la mayoría refirió que la consistencia espesa representa 'más alimento', es adecuada, permite que el niño se llena rápido; los principales tipos de presentación serían las preparaciones blandas, aplastadas y trituradas. Al mismo tiempo, las madres percibieron que el agregado de grasa es útil principalmente para la formación de órganos, también para tener más apetito, completar la cantidad de grasa que el niño recibía cuando tomaba más leche materna y suavizar los alimentos.

Respecto al número y cantidad de comida, para los menores de 1 año la mayoría indicó que deben comer 5 veces al día y en cantidades según su edad y gustos; para los mayores de 1 año, la mayoría percibió que deben comer las veces que quiera y la cantidad va a depender de su apetito y consistencia del alimento.

La mayoría percibía que el consumo diario de frutas y verduras era una práctica adecuada, por contener vitaminas y evitar el estreñimiento, además que deben consumirse de diversos colores. En cuanto a los AOA, la mayoría percibía que deben ser consumidos a diario por ser considerados importantes contra la anemia y para evitar que los niños se enfermen y así, crezcan adecuadamente; pero, deben ser variados para evitar el hostigamiento, excepto el huevo, que podía ser consumido todos los días. Por otro lado, pocas madres pensaban que no era recomendable el consumo diario de estos alimentos.

Muchas de las madres percibieron que durante un episodio de diarrea se debe dar abundantes líquidos para evitar la deshidratación, porque el niño no acepta otras preparaciones; además, mencionaron que se debe brindar alimentos con mayor frecuencia y en menor cantidad, restringiendo menestras, leche, verduras y carnes, como la de gallina. Mientras la mayoría afirmó que después de la enfer- 


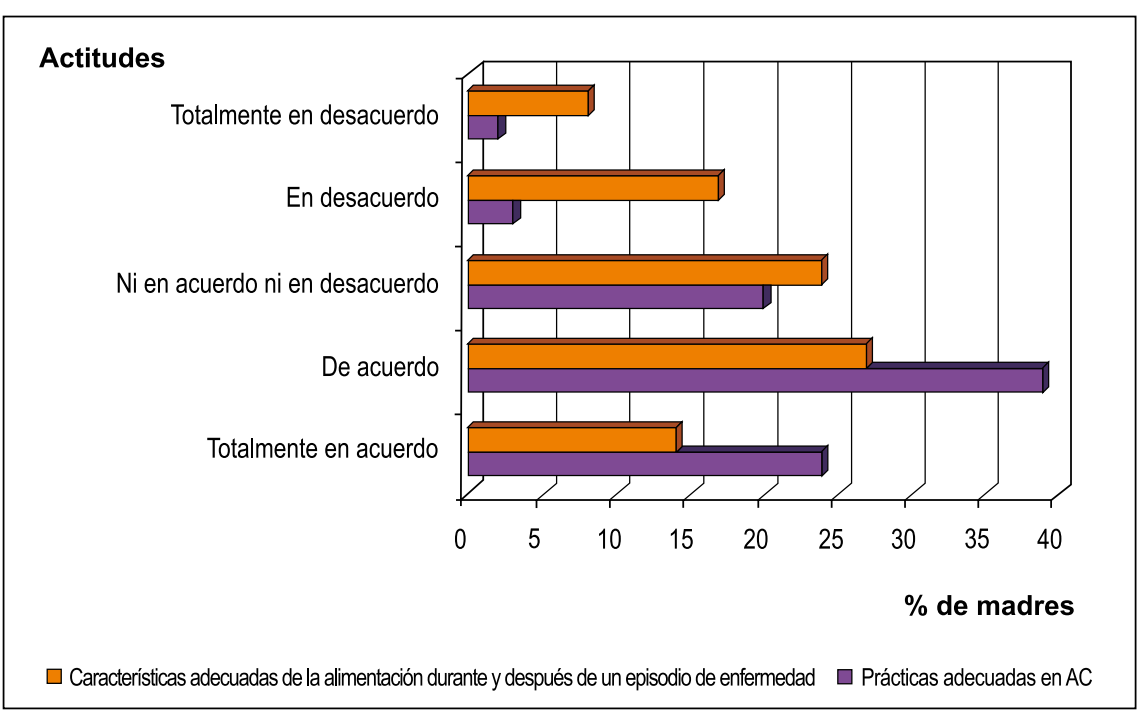

Figura 2. Distribución de madres según su actitud hacia las prácticas adecuadas en alimentación complementaria (AC) y las características adecuadas de la alimentación durante y después de un episodio de enfermedad.

medad los niños deberían comer preparaciones de su consumo habitual según su tolerancia, un menor número de madres dijeron que deben comer preparaciones 'suaves', como caldos y mazamorras, y solo algunas mencionaron 'lo que al niño le guste'.

\section{DISCUSIÓN}

Los resultados de nuestro estudio no pueden ser extrapolados hacia la población, por ser la muestra no probabilística y porque no fue propósito de este estudio que tiene un componente cualitativo; sin embargo, destacamos como fortaleza el tamaño de la muestra para la fase cuantitativa, y el muestreo socioestructural y triangulación para la cualitativa.

El alto nivel de conocimientos hallados sobre alimentación infantil (81\%), fue diferente a lo encontrado por Díaz y col. ${ }^{(13)}$, en el cual solo 4,6\% de las gestantes presentó este nivel de conocimientos. Es preciso mencionar, como parte de la explicación de estos resultados, que las madres del presente estudio participaron en años anteriores de algunas sesiones educativas sobre nutrición brindadas por la ONG WVI.

La LM es el mejor alimento que puede recibir un recién nacido ${ }^{(5)}$; en nuestro estudio, $82 \%$ de las madres respondió adecuadamente sobre su duración. Las madres percibían que la LM se debe mantener hasta los 2 años, porque ayuda a que el niño crezca sano. Al respecto, Alvarado y col. hallaron que algunas madres reconocían que ayuda al desarrollo temprano del niño ${ }^{(14)}$; en otro estudio, se dijo que la LM ofrece al niño mejor nutrición, crecimiento y desarrollo, y crea un vínculo afectivo madre-hijo ${ }^{(15)}$. Esto refleja que los conocimientos de la mayoría de madres del presente estudio eran adecuados y sus percepciones concordaban con ello. Sin embargo, también encontramos que un grupo de madres consideraban que la LM debe suspenderse antes de los dos años, porque el niño no quiere comer otros alimentos, por el dolor al amamantar y por razones laborales. Algo similar se encontró en otros estudios: la imposibilidad de acceder al empleo, dar de lactar daña los senos ${ }^{(15,16)}$, pero no por considerar a la leche materna con poco valor nutricional ${ }^{(17)}$. Esto motivaría a incluir en las intervenciones las técnicas de conservación de la leche materna para brindarla al niño mientras la madre trabaja y así la actividad laboral no sea un obstáculo.

Más de $90 \%$ conocía que la $\mathrm{AC}$ se inicia a los 6 meses, semejante a lo encontrado por Ávila y col. ${ }^{(18)}$, en $87,3 \%$ de las madres. Las madres de nuestro estudio consideraban que a partir de los 6 meses el niño necesita otros alimentos y empieza a tener más apetito; no obstante, un pequeño grupo manifestó que se debe iniciar antes por los antojos del niño al ver comer a otros miembros de la familia. Esto también fue observado en un estudio realizado en nuestro país, en el cual las madres de diferentes regiones tienen razones distintas para decidir la edad de inicio de $\mathrm{AC}$, pues en muchos casos interpretan cualquier gesto del niño como ponerse inquieto o mover sus labios como si les pidiera comida ${ }^{(19)}$. Con respecto a la consistencia de las comidas, deberían ser espesas como puré, mazamorras y comidas aplastadas, pues representa 'más alimento', según lo percibían casi todas las madres. Otro estudio halla que la sopa de pescado, el fríjol y las frutas, como papaya, guayaba y mango, son preferidas por las madres para el inicio de la AC y las menos preferidas las papillas y el huevo ${ }^{(14)}$.

El 63\% de las madres mostró actitudes favorables de AC como el consumo diario de AOA, especialmente el huevo, y así lo percibían para que los niños no se enfermen, evitar la anemia y para su crecimiento adecuado. Murillo halló que más de la mitad de las madres reconocía que los AOA eran de alto valor nutritivo ${ }^{(12)}$. Sin embargo, encontramos que un menor grupo de madres del presente estudio consideraban que no era recomendable su consumo diario. Esto refleja que aún hay temas que se debe seguir trabajando y reforzando para mejorar los conocimientos y actitudes, de tal manera que sus percepciones se modifiquen y estas puedan incidir a mediano o largo plazo en cambios de prácticas de alimentación.

Las madres también percibían que el consumo de frutas y verduras debe ser diario, por sus beneficios, como evitar el estreñimiento y aportar vitaminas, aspecto positivo para promocionar el consumo de estos alimentos desde temprana edad, porque en nuestro país su consumo es bajo, incluso en lugares donde la producción es alta ${ }^{(19)}$. La misma tendencia se observa en Honduras, donde los alimentos menos consumidos por los niños de 6 a 9 meses son los vegetales de 
hojas verdes oscuras y las verduras ricas en vitamina $\mathrm{A}$, como las zanahorias, mango y papayas ${ }^{(18)}$.

Las madres consideraban que se debía brindar al niño 5 comidas al día a partir del año, como lo recomienda el CENAN ${ }^{(20)}$, pero la cantidad será dependiendo de lo que pida o desee el niño; lo que preocupa, pues lo que el niño desea -ya sea poco o bastante- las madres lo consideraban adecuado y no insistían en que terminen su comida, incluso percibían que si les exigían podrían causarles alguna enfermedad.

Durante episodios de diarrea, las madres brindarían abundantes líquidos a sus niños para evitar la deshidratación, comidas más frecuentes pero en menor cantidad, y restringirían ciertos alimentos, como menestras, leche, verduras y carne de gallina. En México, un estudio mostró que más de la mitad de las madres restringía parcialmente la alimentación a sus niños durante episodios de diarrea, pero ofrecía algunos alimentos durante la enfermedad; no obstante, el resto los mantenía en ayuno ${ }^{(21)}$. Esto no fue mencionado por nuestras participantes, pues consideraban que aunque el niño se encontrara con diarrea, siempre debían brindar alimentos o LM a sus niños durante ese proceso.

En términos generales, las madres presentaron conocimientos adecuados. No obstante, las actitudes sobre estos temas serían un aspecto que se debe seguir trabajando, pues aunque no en muchas madres, se pudo observar que aún existen actitudes no favorables influenciadas por sus percepciones erróneas. Para ello, se debe buscar métodos que las motiven, de tal manera que sus actitudes se tornen favorables.

Finalmente, las percepciones que deben ser trabajadas son relacionadas al consumo diario de AOA, cantidad de comida que el niño debe consumir y alimentación del niño durante episodios de enfermedad.

\section{REFERENCIAS BIBLIOGRÁFICAS}

1. ESPGHAN Committee on Nutrition. Complementary feeding: A commentary by the ESPGHAN Committee on Nutrition. J Pediatr Gastroenterol Nutr 2008;46:99-110.

2. Gupta A, Dadhich JP, Faridi MMA. Breastfeeding and Complementary Feeding as a Public Health Intervention for Child Survival in India. Indian J Pediatr [publicación periódica en línea] 2010. January; Se encuentra en: URL:http://www.bpni. org/Article/child-survival-in-India-II.pdf

3. Cereceda MP. Dietética de la teoría a la práctica. Alimentación en las diferentes etapas de la vida. 1ra Edición. Fondo Editorial de la UNMSM. Lima. 2008

4. Organización Panamericana de Salud/Organización Mundial de Salud. Principios de Orientación para la Alimentación Complementaria del Niño Amamantado. Washington DC. 2003.

5. Flores S, Martínez G, Toussaint G, Adell A, Copto A. Alimentación complementaria en los niños mayores de seis meses de edad. Bases técnicas. Bol Med Hosp Infant Mex. 2006;63(2):129-44.

6. Quesada D. Técnica de investigación cuantitativa. Escala de actitudes tipo Likert. Licenciatura en docencia. Investigación Educativa. Lima. Universidad Nacional Mayor de San Marcos. 2006.

7. Gómez A. Percepciones y conocimientos de los actores políticos locales de la cuenca del rió casma en temas de nutrición y seguridad alimentaria. Ancash, enero-junio, 2007.

8. Pineda F, Agudelo CA. Percepciones, actitudes y prácticas en malaria en el Amazonas Colombiano. Rev Salud Pública (Bogotá). 2005;7(3):339348.

9. INEl. (Internet) Perú. Encuesta Demográfica y de Salud Familiar 2007-2008. (Acceso 17 de Diciembre 2009). Tipo de Alimentación Complementaria según meses desde el Nacimiento. Disponible en: http://desa.inei.gob.pe/endes/ endes2007/Publicación\%20ENDES.html

10. World Vision International. Línea de Base del PDA El Salvador. Lima, Perú. 2006.

11. Suazo R. Aplicación de Ensayos Domiciliarios para evaluar la adopción de Prácticas Mejoradas de Alimentación Complementaria. Centro Materno Infantil Juan Pablo II Villa El Salvador, Lima. [Tesis licenciatura]. Lima. Universidad Nacional Mayor de San Marcos; 2007.

12. Murillo S, Brenes H. Prácticas y creencias en nutrición y salud de madres rurales costarricenses. Instituto de Investigación en Salud (INISA), Universidad de Costa Rica.
13. Díaz A, Lugo R, Mendez M, Ramirez M, Reyes J, Torres B. Evaluación del nivel de conocimiento sobre alimentación infantil y algunos factores sociodemográficos asociados al aprendizaje a través del diseño y ejecución de un taller dirigido a las mujeres pertenecientes al club de embarazadas. Ambulatorio Urbano tipo I "Nuevo Barrio". Barquisimeto estado Lara. Junio-diciembre del 2006.

14. Alvarado BE, Tabares RE, Delisle H, Zunzunegui MV. Creencias maternas, prácticas de alimentación y estado nutricional en niños Afro-Colombianos. Archivos Latinoamericanos de Nutrición. 2005;55(1):55-63.

15. Díaz C, Cabrera G, Mateus JC. Representaciones de lactancia en un grupo de mujeres de Cali. Colomb Med. 2003;34:119-23.

16. Sáenz ML, Camacho AE. Prácticas de lactancia materna y alimentación complementaria en un jardín infantil de Bogotá. Rev Salud Pública. 2007;9(4):587-94.

17. Piñeros BS, Camacho NJ. Factores que inciden en la suspensión de la lactancia materna exclusiva. Universidad de los Llanos. Villavicencio - Colombia. Rev Orinoquia. 2004;8(1):6-14.

18. Avila GA, Henne K, Posas J, Thurston A, Acosta ME, Withson D. Conocimientos y prácticas sobre nutrición infantil, enfermedades diarreicas y respiratorias en Lempira, Honduras. Rev Med Hond. 2001;69:52-61.

19. Centro Nacional de Alimentación y Nutrición. Ministerio de Salud, Instituto Nacional de Salud, 2004. Conocimientos, actitudes y prácticas sobre alimentación y nutrición en madres de niños menores de tres años, gestantes y escolares.

20. Instituto Nacional de Salud - Centro Nacional de Alimentación y Nutrición 2004. Lineamientos de Nutrición Materno infantil del Perú. Lima - Perú 2004.

21. Martínez H, Diez S, Meneses LM. Alimentación sostenida durante diarrea aguda en niños menores de cinco años. Salud Pública Mex. 1998;40:141-9.

Manuscrito recibido el 19 de agosto de 2010 y aceptado para publicación el 28 de setiembre de 2010.

Correspondencia:

Mayra Porroa Jacobo

Avenida El Sol 1341 Manzana N Lote 13, Villa El Salvador.

Correo electrónico: mmpj_15 @hotmail.com 